\title{
ANALYSIS OF THE OXIDATION STATE OF PLATINUM PARTICLES IN SUPPORTED CATALYSTS BY DOUBLE DIFFERENTIATION OF XPS LINES
}

\author{
M. Yu. Smirnov ${ }^{1}$, A. V. Kalinkin ${ }^{1}$, E. I. Vovk ${ }^{1,2}$, \\ and V. I. Bukhtiyarov ${ }^{1}$ \\ UDC 544.723.54:544.171.54:546.284-31
}

In the work the double differentiation of functions describing the Pt $4 f_{7 / 2}$ band in the XPS spectra of model supported $\mathrm{Pt} / \mathrm{SiO}_{2}$ catalysts is performed in order to determine the number of different chemical states of platinum particles. The functions for the differentiation are obtained by the deconvolution of the experimental spectral contour into two spin-orbit components. As a result of the performed analysis of the number and position of the minima of the second derivative of the function of $\mathrm{Pt} 4 f_{7 / 2}$ the conditions of the oxidation of platinum particles in the $\mathrm{Pt} / \mathrm{SiO}_{2}$ sample on treating in a $\mathrm{NO}+\mathrm{O}_{2}$ mixture and the reduction of platinum oxide particles on interacting of the $\mathrm{PtO}_{x} / \mathrm{SiO}_{2}$ sample with hydrogen are determined.

DOI: $10.1134 / \mathrm{S} 002247661606010 \mathrm{X}$

Keywords: model catalysts, platinum, silicon dioxide, X-ray photoelectron spectroscopy, differentiation of the spectra.

\section{INTRODUCTION}

The solution of problems associated with the analysis of X-ray photoelectron (XPS) spectra of supported platinum catalysts is difficult due to some problems caused, first of all, by a very low content of platinum. For widely used aluminoplatinum catalysts, the problem of a low platinum concentration is more complicated due to the overlap of the Pt $4 f$ photoemission line (as most suitable for the analysis) with the intense Al $2 p$ line of the support, thus often giving incorrect results. The contribution of the A1 $2 p$ line to the general spectral contour can be considered by calculating the line parameters from the parameters of another line of aluminum (Al2s) using the known relations between the parameters [1]. Another approach to the solution of this problem is to use the Pt $3 d_{5 / 2}$ platinum line for the analysis. This line does not overlap with aluminum lines [2-4], but this line can be recorded only using harder radiation, for example, $\mathrm{Ag} L_{\alpha}(h v=2984.3 \mathrm{eV})$, which is not always available.

The analysis of the Pt $4 f$ spectra of platinum catalysts is also complicated, even when platinum lines do not overlap with the lines of other elements contained in catalysts. The binding energy $E_{\mathrm{b}}\left(\mathrm{Pt} 4 f_{7 / 2}\right)$ is the most important parameter considered in the analysis of the Pt $4 f$ spectra, and it depends on the degree of platinum oxidation and the size of supported particles and their chemical environment. The dependence on the particle size is explained by the final state effect, when a positive hole induced by photoemission on the $4 f$ level in the particles is shielded less efficiently than in bulk metal [5]. Due

${ }^{1}$ Boreskov Institute of Catalysis, Siberian Branch, Russian Academy of Sciences, Novosibirsk, Russia; smirnov@catalysis.ru. ${ }^{2}$ Chemistry Department, Bilkent University, Bilkent, Ankara, Turkey. Translated from Zhurnal Strukturnoi Khimii, Vol. 57, No. 6, pp. 1188-1194, July-August, 2016. Original article submitted September $12,2015$. 
$\mathrm{Al}_{2} \mathrm{O}_{3}$ and $\mathrm{SiO}_{2}$ and other non-conducting supports, towards higher binding energies relative to the peak position of bulk metal can reach $\sim 1.5 \mathrm{eV}[5]$.

The shape of Pt $4 f_{7 / 2}$ and $\mathrm{Pt} 4 f_{5 / 2}$ lines also depends on the chemical state of platinum. The asymmetric shape with a "tail" elongating towards higher binding energies is typical of bulk metallic platinum. The asymmetry is caused by the features of the interaction of photoelectrons with a hole on the core level at a high concentration of electrons in the conduction band near the Fermi level [6]. The shape of the photoemission line can be described by the function derived in [7]. For supported platinum nanoparticles, the lines show the similar asymmetry [8,9], although the form of the function describing photoemission lines in this case can differ from that obtained for bulk metal. At the transition of platinum particles from the metallic state to the oxide state, the Pt $4 f$ lines become symmetric [9]. The symmetry of the lines correlates with the electrophysical properties of platinum oxides. Thus, PtO oxide is known to be a $p$-type semiconductor [11], and according to the data of the ultraviolet photoelectron spectroscopy, its electron density in the conduction band is sufficiently lower than that of metallic platinum [12].

Owing to the above, it is clear that when the Pt $4 f$ spectra of the platinum catalyst cannot be described by one doublet of the symmetric Pt $4 f_{7 / 2}$ and Pt $4 f_{5 / 2}$ lines, it is often difficult to determine the chemical state of platinum particles. Thus, the observed asymmetry can be due either to the existence of platinum particles in two different chemical states (metallic and oxide states) or to the presence of only one type of metallic particles in the studied sample of the catalyst.

In the present work, for the determination of the number of different chemical states of platinum particles in the samples of model $\mathrm{Pt} / \mathrm{SiO}_{2}$ catalysts in their interaction with the gaseous reaction medium, the double numerical differentiation of the function describing the Pt $4 f_{7 / 2}$ line was applied. The double differentiation of XPS spectra has previously been applied for the analysis of spectral contours of $\mathrm{C} 1 s$ and $\mathrm{O} 1 s$ carbon fibers [13], as well as for the determination of the iron distribution over the oxidation states in natural biotite type minerals [14].

\section{EXPERIMENTAL}

Preparation of model samples and measurement of XPS spectra. The preparation of samples of the model catalysts, their treatment in the reaction medium, and the measurement of XPS spectra were carried out on a VG ESCA-3 spectrometer (Great Britain) under the residual pressure in the analytical chamber $<5 \times 10^{-9}$ mbar. The XPS spectra were recorded using $\mathrm{Mg} K_{\alpha}$ non-monochromatic radiation $(h v=1253.6 \mathrm{eV})$ at a voltage of $8.5 \mathrm{kV}$, and a current of $20 \mathrm{~mA}$ on the $\mathrm{X}$-ray tube. The transmission potential of the energy analyzer corresponded to the parameter $\mathrm{HV}=20 \mathrm{~V}$. Before the experiment, the binding energy scale of the spectrometer was calibrated against the positions of lines of gold and copper in the metallic state: $\mathrm{Au} 4 f_{7 / 2}(84.0 \mathrm{eV})$, and $\mathrm{Cu} 2 p_{3 / 2}(932.6 \mathrm{eV})$. The recorded photoemission spectra were processed after the subtraction of the background line approximated by the Shirley function. In order to determine the exact values of the binding energy of the photoemission lines, the $\mathrm{Si} 2 p$ line of silicon dioxide with the binding energy of $103.4 \mathrm{eV}$ was taken as the internal standard. With this calibration method, the binding energy of the $\mathrm{C} 1 s$ line of amorphous carbon accumulated on the sample surface during the spectra measurement varied within $284.8-285.0 \mathrm{eV}$.

The samples of model catalysts were obtained in the preparation chamber of the spectrometer by the procedure described in [9]. Thin films of the $\mathrm{SiO}_{2}$ support were obtained on the surface of tantalum foil by evaporating silicon in

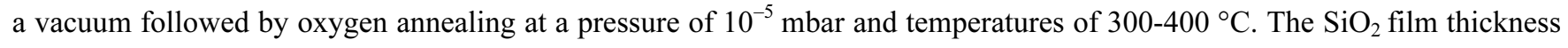
was estimated to be $\sim 10 \mathrm{~nm}$ by reducing the intensity of photoemission lines of the substrate (Ta) during the support formation. Platinum was deposited onto the support surface by thermal evaporation in a vacuum. The platinum content in the samples was determined by the intensity ratio of the Pt $4 f$ and Si2 $p$ photoelectron lines, and taking into account the atomic sensitivity factors, the $\mathrm{Pt} / \mathrm{Si}$ atomic ratios were calculated [15]. The sample was heated by passing the current through the tantalum foil; the temperature was measured by means of the chromel-alumel thermocouple welded on the underside of the foil. 
Analysis of the XPS spectra recorded in the Pt $4 f$ region was started with their deconvolution into two spin-orbit components $\mathrm{Pt} 4 f_{7 / 2}$ and Pt $4 f_{5 / 2}$, using the XPSPeak program [16]. During the deconvolution, it was taken into consideration that the spin-orbit splitting is $3.33 \mathrm{eV}$, and the integrated intensity ratio of the $\mathrm{Pt} 4 f_{7 / 2}$ and $\mathrm{Pt} 4 f_{5 / 2}$ components is $4: 3$ [15]. The $\operatorname{Pt} 4 f_{7 / 2}$ and $\mathrm{Pt} 4 f_{5 / 2}$ lines were supposed to have the same width (FWHM). The asymmetry parameters set by the program to define the shape of the spectral line, were supposed to have the same values for Pt $4 f_{7 / 2}$ and Pt $4 f_{5 / 2}$. The deconvolution of the spectral contour in the Pt $4 f$ region resulted in two smooth functions formally describing the spin-orbit components $\mathrm{Pt} 4 f_{7 / 2}$ and Pt $4 f_{5 / 2}$. Fig. $1 a$ shows the deconvolution of the Pt $4 f$ spectra of the model $\mathrm{Pt} / \mathrm{SiO}_{2}$ sample. Further, the function describing a more intense Pt $4 f_{7 / 2}$ component was used for the analysis.

The function describing the Pt $4 f_{7 / 2}$ line undergoes the operations listed below, and the results of these operations are shown in Fig. $1 b$ :

1. Smoothing of the Pt $4 f_{7 / 2}$ function by five neighboring points using the Origin 6.1 program (Fig. 1b, curve 1 );

2. Numerical differentiation of the smoothed Pt $4 f_{7 / 2}$ function;

3. Smoothing of the first derivative (Fig. $1 b$, curve 2);

4. Differentiation of the smoothed first derivative;

5. Smoothing of the second derivative (Fig. 1b, curve 3).

When the above mentioned operations are carried out, curve 3 is obtained, which is characterized by the minimum whose position corresponds to the peak maximum of the $\mathrm{Pt} 4 f_{7 / 2}$ photoemission line. The two zero values of function 3 , located on either side of the minimum, correspond to the maximum and minimum of first derivative 2 , and to the inflection points on the rising edge and falling edge (on the upgrade and downgrade) of function 1 describing the photoemission line. The numerical value of the local maximum located to the left of the minimum (corresponds to the rising edge of the function) is higher than that of another maximum located to the right of the minimum point (corresponds to falling edge), which is due to the asymmetric shape of the Pt $4 f_{7 / 2}$ spectral line having a "tail" elongating towards the region of higher binding energies.

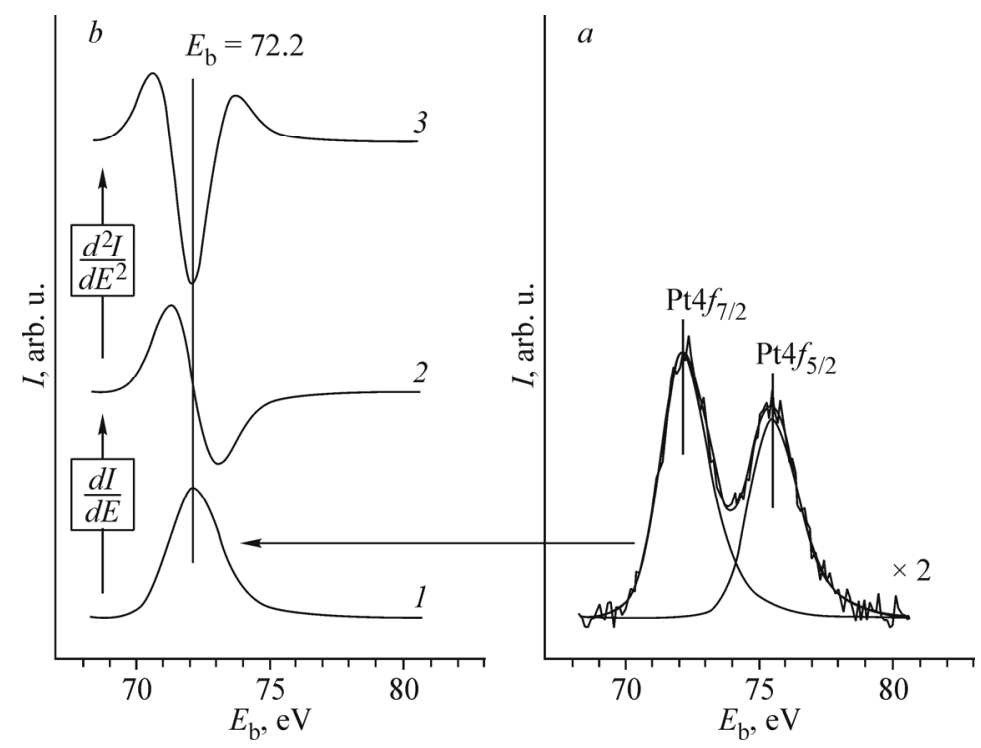

Fig. 1. Deconvolution of the $\mathrm{Pt} 4 f$ spectra of the $\mathrm{Pt} / \mathrm{SiO}_{2}$ sample $(\mathrm{Pt} / \mathrm{Si}$ atomic ratio 0.1$)$ into spin-orbit components $(a)$, step-by-step conversion of the function describing the Pt $4 f_{7 / 2}$ line $(b)$ : smoothing (1), differentiation+smoothing of the derivative (2), repeated differentiation+ smoothing of the second derivative (3). 


\section{RESULTS AND DISCUSSION}

$\mathrm{Pt} / \mathrm{SiO}_{2}+\left(\mathrm{NO}+\mathrm{O}_{2}\right)$. The ease of the investigation of the platinum state in the given system by the XPS method is due to the fact that the Pt $4 f$ line does not overlap with lines of other elements participating in the reaction. Moreover, when the $\mathrm{Pt} / \mathrm{SiO}_{2}$ system interacts with a mixture of $\mathrm{NO}$ and oxygen, no nitrates and nitrites are formed on the surface of the support $\left(\mathrm{SiO}_{2}\right)$, while the supported platinum particles change their chemical state [9].

Fig. $2 a$ shows a set of the spectra recorded in the Pt $f f$ region, when the sample with the atomic ratio $\mathrm{Pt} / \mathrm{Si} \sim 0.3$ interacts with a mixture of $32 \mathrm{mbar} \mathrm{NO}+32 \mathrm{mbar}_{2}$ at different temperatures. The treatment of the sample in the reaction medium was carried out in the preparation chamber of the electron spectrometer, then the sample (without any contact with the atmosphere) was brought into the analytical chamber to collect spectra. Spectrum 1 corresponding to the initial state of the sample before the reaction is presented by a doublet of asymmetric lines whose shape is typical of platinum in the metallic state. The binding energy $E_{\mathrm{b}}\left(\mathrm{Pt} 4 f_{7 / 2}\right)$ is $71.6 \mathrm{eV}$ and noticeably differs from the value of the binding energy for bulk metallic platinum being $71.2 \mathrm{eV}$. Nevertheless, taking into account the method of the samples preparation and the asymmetric shape of the lines in the Pt $4 f$ spectrum, it should be admitted that platinum is in the metallic state. The observed shift in the binding energy of Pt $4 f_{7 / 2}$ relative to the tabular value for metallic platinum can be explained by the final state effect due to the fact that the positive hole appearing under photoemission on the $4 f$ level in the particles is being welded not so effective as in bulk metal; at the same time, the smaller the particle size, the greater the shift value is [5]. The procedure of the double differentiation of the function describing the Pt $4 f_{7 / 2}$ line results in curve 1 shown in Fig. $2 b$. Curve 1 is characterized by one minimum whose position at $71.6 \mathrm{eV}$ is in good agreement with the position of the maximum of the $\mathrm{Pt} 4 f_{7 / 2}$ photoemission line.

After the reaction at $200{ }^{\circ} \mathrm{C}$ and $300{ }^{\circ} \mathrm{C}$, the Pt $f f$ lines are shifted to the region of higher binding energies by $1.6 \mathrm{eV}$ (Fig. $2 a$, spectra 2 and 3). At the same time, the spin-orbit components in the spectrum remain asymmetric. The second derivatives obtained from the Pt $4 f_{7 / 2}$ lines and shown in Fig. $2 b$ as curves 2 and 3, still have only one minimum. This result gives grounds to consider the platinum particles present only in one chemical state. The positions of the maximum of the photoemission line and the minimum in the curve of its second derivative coincide. Based on the results obtained previously $[9,10]$, this state characterized by an increased value of $E_{\mathrm{b}}\left(\mathrm{Pt} 4 f_{7 / 2}\right)$ and the asymmetric shape of the spin-orbit Pt $4 f_{7 / 2}-\mathrm{Pt} 4 f_{5 / 2}$ components, can be interpreted as metallic platinum particles containing dissolved oxygen atoms.
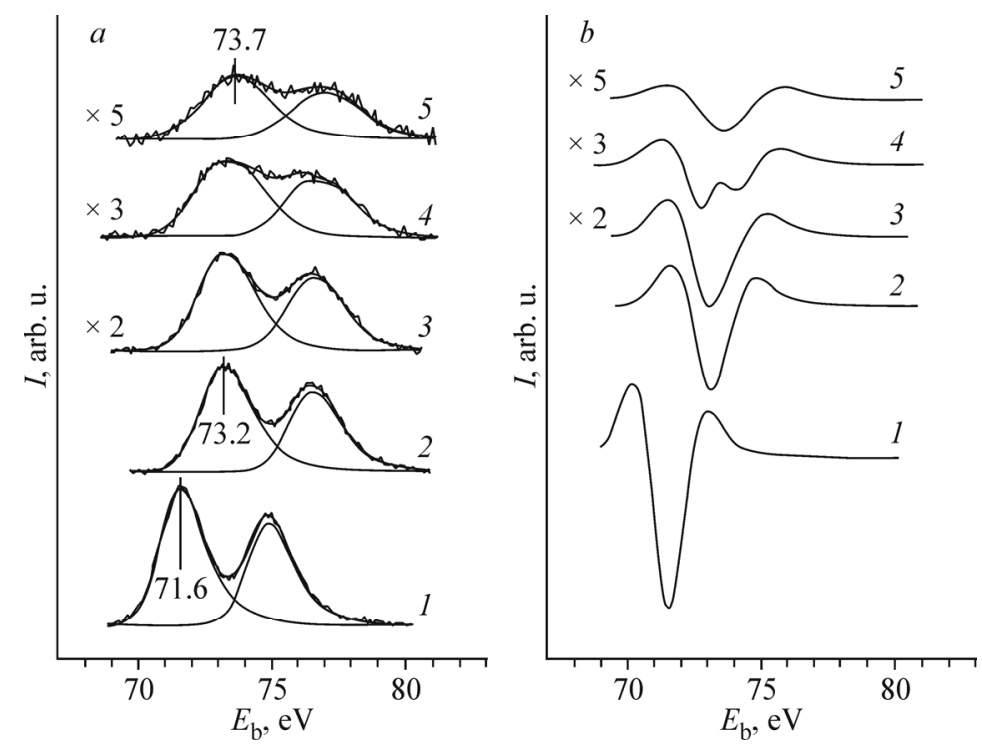

Fig. 2. Pt $4 f$ spectra $(a)$ and second derivatives of the Pt $4 f_{7 / 2}$ functions (b) for the $\mathrm{Pt} / \mathrm{SiO}_{2}$ sample $(\mathrm{Pt} / \mathrm{Si}$ atomic ratio 0.3$)$ in the initial state (1) and after the interaction with a mixture of $32 \mathrm{mbar} \mathrm{NO}+32 \mathrm{mbar}_{2}$ at $200{ }^{\circ} \mathrm{C}(2), 300{ }^{\circ} \mathrm{C}(3), 400{ }^{\circ} \mathrm{C}(4)$, and $500{ }^{\circ} \mathrm{C}(5)$. 
An increase in the reaction temperature up to $400{ }^{\circ} \mathrm{C}$ leads to a pronounced broadening of the line (Fig. $2 a$, spectrum 4). In this case, the shape of the Pt $4 f_{7 / 2}$ photoemission line changes so that its second derivative shows two minima (Fig. 2b, curve 4). This is an important argument to support the idea that after the reaction carried out at this temperature, there are two different chemical types of Pt-containing particles on the surface of the support. Based on the result obtained, spectrum 4 was decomposed into two doublet lines with $E_{\mathrm{b}}\left(\mathrm{Pt} 4 f_{7 / 2}\right)$ being $72.5 \mathrm{eV}$ and $73.8 \mathrm{eV}$ (Fig. 3). The line with a lower value of the binding energy, which is characterized by the asymmetric shape of its spin-orbit components, as in the previous case, can be attributed to metallic platinum particles containing dissolved oxygen. The second line with the symmetric shape

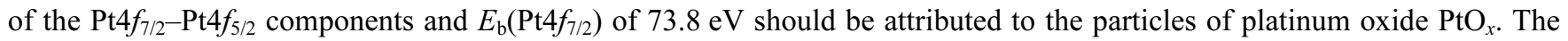
value of the binding energy found for this type of particles is intermediate between the values characteristic of bulk platinum oxides $\mathrm{PtO}$ and $\mathrm{PtO}_{2}$ [17-20].

A further increase in the reaction temperature up to $500{ }^{\circ} \mathrm{C}$ gives spectrum 5 consisting of one doublet whose spinorbit components are of the symmetric shape, and $E_{\mathrm{b}}\left(\mathrm{Pt} 4 f_{7 / 2}\right)$ is noticeably higher than the value typical of bulk metallic platinum. The second derivative (Fig. 2b, curve 5) represents a function with one minimum, symmetric relative to the axis running through this minimum. This is the basis to suppose that after the treatment, the samples contain only $\mathrm{PtO}_{x}$ particles.

$\mathbf{P t O}_{x} / \mathrm{SiO}_{2}+\mathbf{H}_{2}$. The hydrogen reduction of platinum oxide particles whose formation is described above, was performed under a hydrogen pressure of 16 mbar and temperatures ranging from room to $200{ }^{\circ} \mathrm{C}$. Fig. $4 a$ shows the Pt $4 f$ spectra obtained when the $\mathrm{PtO}_{x} / \mathrm{SiO}_{2}$ sample interacts with hydrogen, and Fig. $4 b$ shows the corresponding second derivatives of the functions describing the Pt $4 f_{7 / 2}$ line. Spectrum 1 in Fig. $4 a$ and its second derivative (Fig. $4 b$, curve 1 ) are attributed to the initial state of the sample before the reaction, in which platinum particles are in the oxidized state. This spectrum and the corresponding second derivative are taken from Fig. 2 (curves 5).

After the interaction with hydrogen at $50{ }^{\circ} \mathrm{C}$, the spectral lines in the $\mathrm{Pt} 4 f_{7 / 2}-\mathrm{Pt} 4 f_{5 / 2}$ doublet are broadened, the lines are shifted towards the region of lower binding energies. At the same time, the spin-orbit Pt $4 f_{7 / 2}$ component adopts such a shape that its second derivative has two minima at $73.1 \mathrm{eV}$ and $74.3 \mathrm{eV}$, which indicates the presence of two states of platinum in the sample. This is the basis to decompose the Pt $4 f$ spectrum into two doublets as shown in Fig. 5 (spectrum 1 ). It is obvious that the doublet with a higher value $E_{\mathrm{b}}\left(\mathrm{Pt} 4 f_{7 / 2}\right)$ corresponds to platinum oxide particles, and another doublet formed by the asymmetric lines with a characteristic "tail" corresponds to metallic platinum particles containing dissolved oxygen. With an increase in the reaction temperature up to $100^{\circ} \mathrm{C}$, the $\mathrm{Pt} 4 f$ spectrum is still described by two doublet lines; at the same time the contribution of metallic platinum increases due to a decrease in the contribution of platinum oxide (Fig. $4 b$,

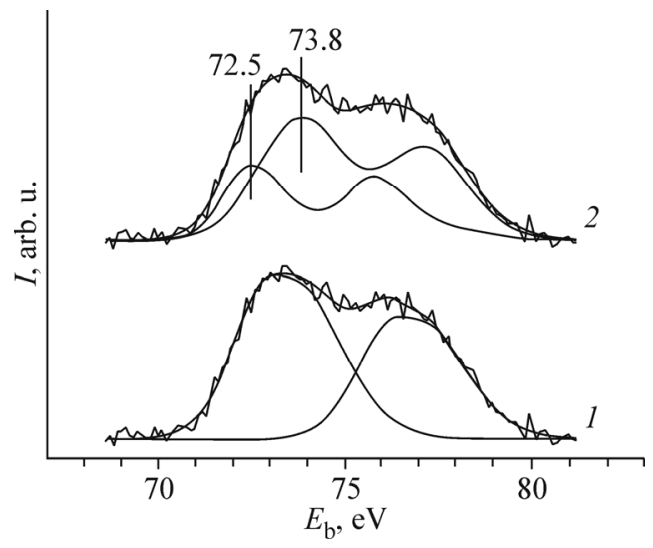

Fig. 3. $\mathrm{Pt} 4 f$ spectrum of the $\mathrm{Pt} / \mathrm{SiO}_{2}$ sample $(\mathrm{Pt} / \mathrm{Si}$ atomic ratio 0.3$)$ after the interaction with a mixture of 32 mbar $\mathrm{NO}+32 \mathrm{mbar}_{2}$ at $400{ }^{\circ} \mathrm{C}(1)$ and its deconvolution into two doublets (2). 

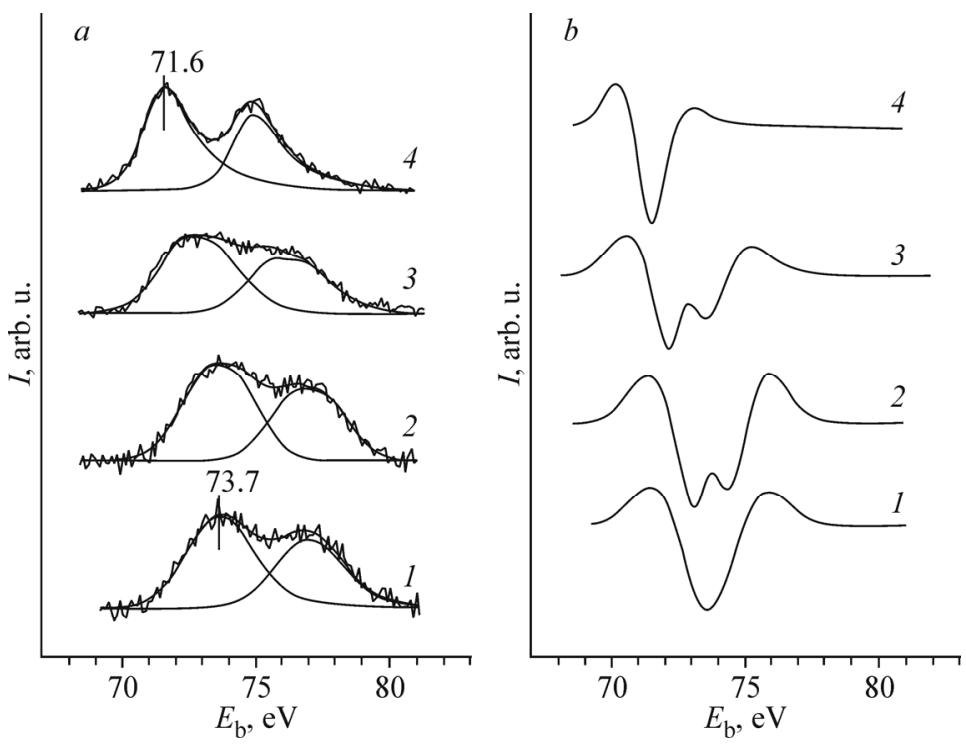

Fig. 4. Pt $4 f(a)$ spectra and second derivatives of the Pt $4 f_{7 / 2}$ functions (b) for the $\mathrm{PtO}_{x} / \mathrm{SiO}_{2}$ sample in the initial state $(1)$ and after the interaction with 16 mbar hydrogen at $50{ }^{\circ} \mathrm{C}(2), 100{ }^{\circ} \mathrm{C}(3)$, and $200{ }^{\circ} \mathrm{C}(4)$.

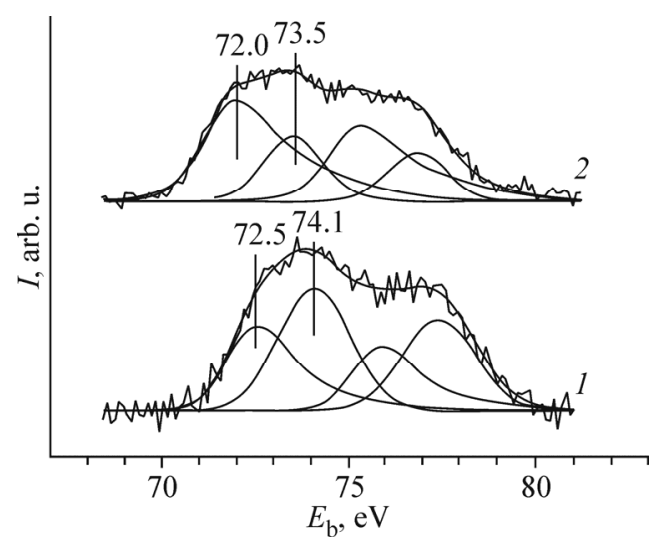

Fig. 5. Pt $4 f$ spectral decomposition of the $\mathrm{PtO}_{x} / \mathrm{SiO}_{2}$ sample into two doublets after the interaction with 16 mbar hydrogen at $50{ }^{\circ} \mathrm{C}(1)$ and $100{ }^{\circ} \mathrm{C}(2)$.

curve 3, and Fig. 5, curve 2). Platinum oxide particles are completely reduced to metal after hydrogen treatment at $200{ }^{\circ} \mathrm{C}$. Under these conditions, the second derivative of the Pt $4 f_{7 / 2}$ line has only one minimum, and the corresponding Pt $4 f$ spectrum can be described by one doublet with $E_{\mathrm{b}}\left(\mathrm{Pt} 4 f_{7 / 2}\right)$ being $71.6 \mathrm{eV}$.

\section{CONCLUSIONS}

This paper descibes the application of the double differentiation of the function describing the $\operatorname{Pt} 4 f_{7 / 2}$ spectral line for the correct determination of the number of different platinum states in the samples of model $\mathrm{Pt} / \mathrm{SiO}_{2}$ catalysts during the oxidation-reduction interaction with the reaction medium. As a result of the performed analysis of the spectra, it is determined that with an increase in the interaction temperature of $\mathrm{Pt} / \mathrm{SiO}_{2}$ with the $\mathrm{NO}+\mathrm{O}_{2}$ mixture, at the early stage, the dissolution of oxygen atoms occurs in metallic platinum particles. After the treatment at $400{ }^{\circ} \mathrm{C}$, the simultaneous presence of 
two states of platinum (metallic platinum particles with dissolved oxygen and $\mathrm{PtO}_{x}$ platinum oxide particles) is observed. By the reduction of the oxidized sample with hydrogen, the inverse order of the change in the platinum states is observed.

This work was carried out in the framework of the government order for the Boreskov Institute of Catalysis Siberian

Branch, Russian Academy of Sciences. The work was supported by the Council of Grants of the President of Russian Federation (Program for State Support for Leading Scientific Schools of the Russian Federation, Grant NSh-5340.2014.3).

\section{REFERENCES}

1. M. Yu. Smirnov, A. V. Kalinkin, and V. I. Bukhtiyarov, J. Struct. Chem., 48, 1053-1060 (2007).

2. A. V. Kalinkin, M. Yu. Smirnov, A. I. Nizovskii, and V. I. Bukhtiyarov, J. Electron Spectrosc. Relat. Phenom., 177, 15 (2010).

3. O. B. Bel'skaya, T. I. Gulyaeva, V. P. Talsi, et al., Kinet. Catal., 55, 792-798 (2014).

4. R. M. Mironenko, O. B. Bel'skaya, V. P. Talsi, et al., Appl. Catal. A, 469, 472 (2014).

5. M. G. Mason, Phys. Rev. B, 27, 748 (1983).

6. Practical Surface analysis by Auger and X-ray photoelectron spectroscopy, D. Briggs, M. P. Seah (eds.), Mir, Moscow (1987).

7. S. Doniach and M. Šunjič, J. Phys. C: Solid State Phys., 3, 285 (1970).

8. A. Yu. Stakheev, Yu. M. Shulga, N. A. Gaidai, et al., Mendeleev Commun., 5, 186 (2001).

9. M. Yu. Smirnov, E. I. Vovk, A. V. Kalinkin, et al., Kinet. Catal., 53, 117-124 (2012).

10. M. Yu. Smirnov, A. V. Kalinkin, D. A. Nazimov, et al., Kinet. Catal., 56, 540 (2015).

11. J. R. McBride, G. W. Graham, C. R. Peters, and W. H. Weber, J. Appl. Phys., 69, 1596 (1991).

12. T. H. Fleisch, G. W. Zajac, J. O. Schreiner, and G. J. Mains, Appl. Surf. Sci., 26, 488 (1986).

13. A. Proctor and A. Sherwood, Anal. Chem., 54, 13 (1982).

14. S. P. Raeburn, E. S. Ilton, and D. R. Veblen, Geochim. Cosmochim. Acta, 61, 4519 (1997).

15. J. F. Moulder, W. F. Stickle, P. E. Sobol, and K. D. Bomben, Handbook of X-ray Photoelectron Spectroscopy, J. Chastain (ed.), Perkin-Elmer Co (1992).

16. http://www.uksaf.org/xpspeak41.zip.

17. V. K. Kaushik, Z. Phys. Chem., 173, 105 (1991).

18. A. J. Silvestre, E. A. Sepulveda, R. F. Rodriguez, and J. A. Anderson, J. Catal., 223, 179 (2004).

19. S. Zafeiratos, G. Papakonstantinou, M. M. Jacksic, and S. G. Neophytides, J. Catal., 232, 127 (2005).

20. C. H. Huang, I. K. Wang, Y. M. Lin, et al., J. Mol. Catal. A, 316, 163 (2010). 